\title{
Ground State Correlations and Structure of Odd Spherical Nuclei
}

\author{
$\underline{\text { S. Mishev }}^{1,2}$ and V. V. Voronov ${ }^{1}$ \\ 1 Bogoliubov Laboratory of Theoretical Physics, Joint Institute for Nuclear Research, \\ Dubna 141980, Russian Federation \\ 2 Institute for Nuclear Research and Nuclear Energy, Bulgarian Academy of Sciences, \\ Sofia 1784, Bulgaria
}

\begin{abstract}
It is well known that the Pauli principle plays a substantial role at low energies because the phonon operators are not ideal boson operators. Calculating the exact commutators between the quasiparticle and phonon operators one can take into account the Pauli principle corrections. Besides the ground state correlations due to the quasiparticle interaction in the ground state influence the single particle fragmentation as well. In this paper, we generalize the basic QPM equations to account for both mentioned effects. As an illustration of our approach, calculations on the structure of the low-lying states in ${ }^{131} \mathrm{Ba}$ have been performed.
\end{abstract}

\section{Introduction}

In the forthcoming period there will be an increasing activity in the domain of unstable nuclei studies due to the start of operation at several major facilities. A theoretical investigation of odd nuclei far from stability demands to include the ground state correlation effects.

The quasiparticle-phonon nuclear model (QPM) [1] is widely used for the description of the energies and fragmentation of nuclear excitations. The different versions of the QPM equations for odd spherical nuclei are given in [2-4]. It has been shown $[2,5]$ that the Pauli principle plays a substantial role at low energies, but the ground state correlation effects were not taken into account in these calculations. From the other side, the ground state correlations influence the single particle fragmentation [6] as they shift the strength to higher excitation energies.

In this paper, we generalize the basic QPM equations to account for both mentioned effects. We treat long-range ground state correlations by including backwardgoing quasiparticle-phonon vertices using the equation of motion method [7] with explicitly accounting for the Pauli principle. As an illustration of our approach, calculations of the structure of the low-lying states in ${ }^{131} \mathrm{Ba}$ have been performed.

\section{Basic Formulae}

We employ the QPM-Hamiltonian including an average nuclear field described by the Woods-Saxon potential, pairing interactions, isoscalar particle-hole residual forces in separable form with the Bohr-Mottelson radial dependence [8]: 


$$
\begin{aligned}
H=\sum_{\tau}^{(n, p)}\left\{\sum_{j m}\left(E_{j}-\lambda_{\tau}\right) a_{j m}^{\dagger} a_{j m}-\frac{1}{4} G_{\tau}^{(0)}\right. & :\left(P_{0}^{\dagger} P_{0}\right)^{\tau}: \\
& \left.-\frac{1}{2} \sum_{\lambda \mu} \kappa^{(\lambda)}:\left(M_{\lambda \mu}^{\dagger} M_{\lambda \mu}\right):\right\} .
\end{aligned}
$$

The single-particle states are specified by the quantum numbers $(j m) ; E_{j}$ are the single-particle energies; $\lambda_{\tau}$ is the chemical potential; $G_{\tau}^{(0)}$ and $\kappa^{(\lambda)}$ are the strengths in the p-p and in the p-h channel, respectively. The sum goes over protons(p) and neutrons(n) independently and the notation $\tau=\{n, p\}$ is used.The pair creation and the multipole operators entering the normal products in (1) are defined as follows:

$$
\begin{gathered}
P_{0}^{+}=\sum_{j m}(-1)^{j-m} a_{j m}^{+} a_{j-m}^{+}, \\
M_{\lambda \mu}^{+}=\frac{1}{\sqrt{2 \lambda+1}} \sum_{j j^{\prime} m m^{\prime}} f_{j j^{\prime}}^{(\lambda)}\left\langle j m j^{\prime} m^{\prime} \mid \lambda \mu\right\rangle a_{j m}^{+} a_{j^{\prime} m^{\prime}},
\end{gathered}
$$

where $f_{j j^{\prime}}^{(\lambda)}$ are the single particle radial matrix elements of the residual forces.

In what follows we work in quasiparticle representation, defined by the canonical Bogoliubov transformation:

$$
a_{j m}^{+}=u_{j} \alpha_{j m}^{+}+(-1)^{j-m} v_{j} \alpha_{j-m} .
$$

The Hamiltonian can be represented in terms of bifermion quasiparticle operators (and their conjugate ones):

$$
\begin{gathered}
B\left(j j^{\prime} ; \lambda \mu\right)=\sum_{m m^{\prime}}(-1)^{j^{\prime}+m^{\prime}}\left\langle j m j^{\prime} m^{\prime} \mid \lambda \mu\right\rangle \alpha_{j m}^{+} \alpha_{j^{\prime}-m^{\prime}}, \\
A^{+}\left(j j^{\prime} ; \lambda \mu\right)=\sum_{m m^{\prime}}\left\langle j m j^{\prime} m^{\prime} \mid \lambda \mu\right\rangle \alpha_{j m}^{+} \alpha_{j^{\prime} m^{\prime}}^{+} .
\end{gathered}
$$

The phonon creation operators are defined in the two-quasiparticle space in a standard fashion:

$$
Q_{\lambda \mu i}^{+}=\frac{1}{2} \sum_{j j^{\prime}}\left\{\psi_{j j^{\prime}}^{\lambda i} A^{+}\left(j j^{\prime} ; \lambda \mu\right)-(-1)^{\lambda-\mu} \varphi_{j j^{\prime}}^{\lambda i} A\left(j j^{\prime} ; \lambda-\mu\right)\right\},
$$

where the index $\lambda=0,1,2,3, \ldots$ denotes multipolarity and $\mu$ is its $z$-projection in the laboratory system. The normalization of the one-phonon states reads:

$$
\left\langle\left|\left[Q_{\lambda \mu i}, Q_{\lambda^{\prime} \mu^{\prime} i^{\prime}}^{+}\right]\right|\right\rangle=\delta_{\lambda \lambda^{\prime}} \delta_{\mu \mu^{\prime}} \delta_{i i^{\prime}} .
$$

In terms of quasiparticles and phonons the Hamiltonian is rewritten 


$$
\begin{gathered}
H=h_{0}+h_{p p}+h_{Q Q}+h_{Q B}, \\
h_{0}+h_{p p}=\sum_{j m} \varepsilon_{j} \alpha_{j m}^{+} \alpha_{j m}, \\
h_{Q Q}=-\frac{1}{8} \sum_{\lambda \mu i i^{\prime}} \mathcal{A}\left(\lambda i i^{\prime}\right)\left(Q_{\lambda \mu i}^{+}+(-)^{\lambda-\mu} Q_{\lambda-\mu i}\right)\left(Q_{\lambda-\mu i^{\prime}}^{+}+(-)^{\lambda+\mu} Q_{\lambda \mu i^{\prime}}\right), \\
h_{Q B}=-\frac{1}{2 \sqrt{2}} \sum_{\lambda \mu i j j^{\prime}} \frac{\pi_{j}}{\pi_{\lambda}} \Gamma\left(j j^{\prime} \lambda i\right)\left((-)^{\lambda-\mu} Q_{\lambda \mu i}^{+}+Q_{\lambda-\mu i}\right) B\left(j j^{\prime} ; \lambda-\mu\right)+h . c .
\end{gathered}
$$

where

$$
\begin{gathered}
\mathcal{A}\left(\lambda i i^{\prime}\right)=\frac{X^{\lambda i}+X^{\lambda i}}{\sqrt{Y^{\lambda i} Y^{\lambda i^{\prime}}}}, \\
\Gamma\left(j j^{\prime} \lambda i\right)=\frac{\pi_{\lambda}}{\pi_{j}} \frac{v_{j j^{\prime}}^{(-)} f_{j j^{\prime}}^{(\lambda)}}{\sqrt{Y^{\lambda i}}}, \\
X^{\lambda i}=\sum_{j j^{\prime}} \frac{\left(f_{j j^{\prime}}^{(\lambda)} u_{j j^{\prime}}^{(+)}\right)^{2} \varepsilon_{j j^{\prime}}}{\varepsilon_{j j^{\prime}}^{2}-\omega_{\lambda i}^{2}}, \\
Y^{\lambda i}=\sum_{j j^{\prime}} \frac{\left(f_{j j^{\prime}}^{(\lambda)} u_{j j^{\prime}}^{(+)}\right)^{2} \varepsilon_{j j^{\prime}} \omega_{\lambda i}}{\left(\varepsilon_{j j^{\prime}}^{2}-\omega_{\lambda i}^{2}\right)^{2}},
\end{gathered}
$$

with $v_{j j^{\prime}}^{(-)}=u_{j} u_{j^{\prime}}-v_{j} v_{j^{\prime}}$ and $u_{j j^{\prime}}^{(+)}=u_{j^{\prime}} v_{j}+u_{j} v_{j^{\prime}}$. Here and further we use the notation $\pi_{j}=\sqrt{(2 j+1)}$.

The model wave function of an odd spherical nucleus is taken in the form [6]:

$$
\Psi_{\nu}(J M)=O_{J M \nu}^{+}|\rangle
$$

where

$O_{J M \nu}^{+}=C_{J \nu} \alpha_{J M}^{+}+\sum_{i} D_{j}^{\lambda i}(J \nu) P_{j \lambda i}^{+}(J M)-E_{J \nu} \tilde{\alpha}_{J M}-\sum_{i} F_{j}^{\lambda i}(J \nu) \tilde{P}_{j \lambda i}(J M)$,

with

$$
P_{j \lambda i}^{+}(J M)=\left[\alpha_{j m}^{+} Q_{\lambda \mu i}^{+}\right]_{J M}
$$


and $\sim$ stands for time conjugate according to the convention: $\tilde{P}_{j \lambda i}(J M)=$ $(-1)^{J-M} P_{j \lambda i}(J-M)$.

We apply the equation of motion method to the excitation operator (3):

$$
\left\langle\left|\left\{\delta O_{J M \nu}, H, O_{J M \nu}^{+}\right\}\right|\right\rangle=\eta_{J \nu}\left\langle\left|\left\{\delta O_{J M}, O_{J M}^{+}\right\}\right|\right\rangle .
$$

Following the linearization procedure [7], at the final state of calculation of the matrix elements, we consider the ground state to be a vacuum state for both operators $\alpha_{J M}$ and $Q_{\lambda \mu i}$.

In all calculations the exact commutation relations between the quasiparticle and phonon operators are considered:

$$
\left[\alpha_{j m}, Q_{J M \nu}^{+}\right]=\sum_{j^{\prime} m^{\prime}}\left\langle j m j^{\prime} m^{\prime} \mid J M\right\rangle \psi_{j j^{\prime}}^{J \nu} \alpha_{j^{\prime} m^{\prime}}^{+} .
$$

The normalization condition of the wave function reads

$$
\begin{aligned}
& \left\langle\left|\left\{O_{J M \nu}, O_{J M \nu}^{+}\right\}\right|\right\rangle=C_{J \nu}^{2}+E_{J \nu}^{2}+\sum_{j \lambda i}\left[D_{j}^{\lambda i}(J \nu)\right]^{2}+\sum_{j \lambda i}\left[F_{j}^{\lambda i}(J \nu)\right]^{2}+ \\
& +\sum_{j \lambda i j^{\prime} \lambda^{\prime} i^{\prime}}\left[D_{j}^{\lambda i}(J \nu) D_{j^{\prime}}^{\lambda^{\prime} i^{\prime}}(J \nu)+F_{j}^{\lambda i}(J \nu) F_{j^{\prime}}^{\lambda^{\prime} i^{\prime}}(J \nu)\right] \mathcal{L}_{J}\left(j \lambda i \mid j^{\prime} \lambda^{\prime} i^{\prime}\right)=1 .
\end{aligned}
$$

The equation of motion leads to the following system of linear equations for each state with quantum numbers $J M$ :

$$
\begin{gathered}
\left(\begin{array}{cccc}
\varepsilon_{J} & V\left(J j^{\prime} \lambda^{\prime} i^{\prime}\right) & G(J) & -W\left(J j^{\prime} \lambda^{\prime} i^{\prime}\right) \\
V(J j \lambda i) & K_{J}\left(j \lambda i \mid j^{\prime} \lambda i^{\prime}\right) & -W(J j \lambda i) & S_{J}\left(j \lambda i \mid j^{\prime} \lambda^{\prime} i^{\prime}\right) \\
G(J) & -W\left(J j^{\prime} \lambda^{\prime} i^{\prime}\right) & -\varepsilon_{J} & -V\left(J j^{\prime} \lambda^{\prime} i^{\prime}\right) \\
-W(J j \lambda i) & S_{J}\left(j^{\prime} \lambda^{\prime} i^{\prime} \mid j \lambda i\right) & -V(J j \lambda i) & -K_{J}\left(j \lambda i \mid j^{\prime} \lambda i^{\prime}\right)
\end{array}\right)\left(\begin{array}{l}
C_{J \nu} \\
D_{j^{\prime}}^{\lambda^{\prime} i^{\prime}}(J \nu) \\
-E_{J \nu} \\
-F_{j^{\prime}}^{\lambda^{\prime} i^{\prime}}(J \nu)
\end{array}\right)= \\
=\eta_{J \nu}\left(\begin{array}{l}
C_{J \nu} \\
D_{j}^{\lambda i}(J \nu)+\sum_{j^{\prime} \lambda^{\prime} i^{\prime}} D_{j^{\prime}}^{\lambda^{\prime} i^{\prime}}(J \nu) \mathcal{L}_{J}\left(j \lambda i \mid j^{\prime} \lambda^{\prime} i^{\prime}\right) \\
-E_{J \nu} \\
-F_{j}^{\lambda i}(J \nu)-\sum_{j^{\prime} \lambda^{\prime} i^{\prime}} F_{j^{\prime}}^{\lambda^{\prime} i^{\prime}}(J \nu) \mathcal{L}_{J}\left(j \lambda i \mid j^{\prime} \lambda^{\prime} i^{\prime}\right)
\end{array}\right)
\end{gathered}
$$

The average value of $H$ over the wave functions (2) is 


$$
\begin{aligned}
<\Psi_{\nu}^{*} & (J M) H \Psi_{\nu}(J M)>=\left[C_{J \nu}^{2}-E_{J \nu}^{2}\right] \varepsilon_{J} \\
& +\sum_{j \lambda i j^{\prime} \lambda^{\prime} i^{\prime}}\left(D_{j}^{\lambda i}(J \nu) D_{j^{\prime}}^{\lambda^{\prime} i^{\prime}}(J \nu)+F_{j}^{\lambda i}(J \nu) F_{j^{\prime}}^{\lambda^{\prime} i^{\prime}}(J \nu)\right) I_{J}\left(j \lambda i \mid j^{\prime} \lambda^{\prime} i^{\prime}\right) \\
& +2 \sum_{j \lambda i}\left(C_{J \nu} D_{j}^{\lambda i}(J \nu)-E_{J \nu} F_{j}^{\lambda i}(J \nu)\right) V(J j \lambda i) \\
& +2 \sum_{j \lambda i}\left(C_{J \nu} F_{j}^{\lambda i}(J \nu)+E_{J \nu} D_{j}^{\lambda i}(J \nu)\right) W(J j \lambda i) \\
& -2 C_{J \nu} E_{J \nu} G_{J}-2 \sum_{j \lambda i j^{\prime} \lambda^{\prime} i^{\prime}} D_{j}^{\lambda i} F_{j^{\prime}}^{\lambda^{\prime} i^{\prime}} S_{J}\left(j \lambda i \mid j^{\prime} \lambda^{\prime} i^{\prime}\right) .
\end{aligned}
$$

We give the explicit expressions for the quantities entering the above formulas with short comments

$$
\begin{gathered}
\mathcal{L}_{J}\left(j \lambda i \mid j^{\prime} \lambda^{\prime} i^{\prime}\right)=\pi_{\lambda} \pi_{\lambda^{\prime}} \sum_{j_{1}} \psi_{j_{1} j}^{\lambda^{\prime} i^{\prime}} \psi_{j_{1} j^{\prime}}^{\lambda i}\left\{\begin{array}{ccc}
j^{\prime} & j_{1} & \lambda \\
j & J & \lambda^{\prime}
\end{array}\right\}, \\
G_{J}=\left\langle\left|\left\{\tilde{\alpha}_{J M}^{+},\left[H, \alpha_{J M}^{+}\right]\right\}\right|\right\rangle=\sqrt{2} \sum_{\lambda i j} \frac{\pi_{\lambda}}{\pi_{J}} \Gamma(J j \lambda i) \varphi_{J j}^{\lambda i}, \\
V(J j \lambda i)=\left\langle\left|\left\{\left[\alpha_{J M}, H\right], P_{j \lambda i}^{+}(J M)\right\}\right|\right\rangle= \\
=-\frac{1}{\sqrt{2}} \Gamma(J j \lambda i)-\frac{1}{\sqrt{2}} \sum_{j^{\prime} \lambda^{\prime} i^{\prime}}\left(\mathcal{T}_{J}\left(j \lambda i ; j^{\prime} \lambda^{\prime} i^{\prime}\right)+\mathcal{L}_{J}\left(j \lambda i \mid j^{\prime} \lambda^{\prime} i^{\prime}\right)\right) \Gamma\left(J j^{\prime} \lambda^{\prime} i^{\prime}\right) .
\end{gathered}
$$

As a result of the application of the equation of motion method, the matrix elements $V(J j \lambda i)$ between quasiparticle and quasiparticle $\otimes$ phonon states differ by the ones obtained earlier [2] by an additive containing $\mathcal{T}_{J}\left(j \lambda i \mid j^{\prime} \lambda^{\prime} i^{\prime}\right)$

$$
\begin{gathered}
\mathcal{T}_{J}\left(j \lambda i \mid j^{\prime} \lambda^{\prime} i^{\prime}\right)=\pi_{\lambda} \pi_{\lambda^{\prime}} \sum_{j_{1}} \psi_{j_{1} j^{\prime}}^{\lambda i} \varphi_{j_{1} j}^{\lambda^{\prime} i^{\prime}}\left\{\begin{array}{ccc}
j^{\prime} & j_{1} & \lambda \\
j & J & \lambda^{\prime}
\end{array}\right\} . \\
W(J j \lambda i)=\left\langle\left|\left\{\left[\alpha_{J M}^{+}, H\right], \tilde{P}_{j \lambda i}^{+}(J M)\right\}\right|\right\rangle= \\
=-\frac{1}{4} \frac{\pi_{\lambda}}{\pi_{J}} \sum_{i^{\prime} \tau_{0}} \mathcal{A}_{\tau_{0}}\left(\lambda i i^{\prime}\right) \varphi_{J j}^{\lambda i^{\prime}} \\
-\frac{1}{4} \sum_{\lambda^{\prime} j^{\prime} i^{\prime} i^{\prime \prime} \tau_{0}} \mathcal{A}_{\tau_{0}}\left(\lambda^{\prime} i^{\prime} i^{\prime \prime}\right) \frac{\pi_{\lambda^{\prime}}}{\pi_{J}}\left[\varphi_{J j^{\prime}}^{\lambda^{\prime} i^{\prime}} \mathcal{L}_{J}\left(j \lambda i \mid j^{\prime} \lambda^{\prime} i^{\prime \prime}\right)-\psi_{J j^{\prime}}^{\lambda^{\prime} i^{\prime \prime}} \mathcal{T}_{J}\left(j \lambda i \mid j^{\prime} \lambda^{\prime} i^{\prime}\right)\right] .
\end{gathered}
$$

The matrix elements $W(J j \lambda i)$ appear after the inclusion of the backward-going terms in the operator (3) and they present a central issue of this work. 


$$
\begin{gathered}
S_{J}\left(j \lambda i \mid j^{\prime} \lambda^{\prime} i^{\prime}\right)=\left\langle\left|\left\{\tilde{P}_{j \lambda i}^{+}(J M),\left[H, P_{j^{\prime} \lambda^{\prime} i^{\prime}}^{+}(J M)\right]\right\}\right|\right\rangle=-G_{j^{\prime}} \mathcal{T}_{J}\left(j \lambda i \mid j^{\prime} \lambda^{\prime} i^{\prime}\right), \\
I_{J}\left(j \lambda i \mid j^{\prime} \lambda^{\prime} i^{\prime}\right)=\left\langle\left|\left\{P_{j \lambda i}(J M),\left[H, P_{j^{\prime} \lambda^{\prime} i^{\prime}}^{+}(J M)\right]\right\}\right|\right\rangle, \\
I_{J}\left(j \lambda i \mid j^{\prime} \lambda^{\prime} i^{\prime}\right)+I_{J}\left(j^{\prime} \lambda^{\prime} i^{\prime} \mid j \lambda i\right)=2 \delta_{j j^{\prime}} \delta_{\lambda \lambda^{\prime}} \delta_{i i^{\prime}}\left(\omega_{\lambda i}+\varepsilon_{j}\right)+ \\
+\mathcal{L}_{J}\left(j^{\prime} \lambda^{\prime} i^{\prime} \mid j \lambda i\right)\left(\varepsilon_{j^{\prime} j}+\omega_{\lambda^{\prime} i^{\prime}}+\omega_{\lambda i}\right)-\mathcal{R}_{J}\left(j \lambda i \mid j^{\prime} \lambda^{\prime} i^{\prime}\right), \\
\mathcal{R}_{J}\left(j \lambda i \mid j^{\prime} \lambda^{\prime} i^{\prime}\right)= \\
=\frac{1}{4} \sum_{i_{1} \tau_{0}}\left[\mathcal{A}_{\tau_{0}}\left(\lambda i_{1} i\right) \mathcal{L}_{J}\left(j^{\prime} \lambda^{\prime} i^{\prime} \mid j \lambda i_{1}\right)+\mathcal{A}_{\tau_{0}}\left(\lambda^{\prime} i_{1} i^{\prime}\right) \mathcal{L}_{J}\left(j \lambda i \mid j^{\prime} \lambda^{\prime} i_{1}\right)\right]+ \\
+\frac{1}{4} \sum_{\lambda_{1} i_{1} i_{2} j_{1} \tau_{0}} \mathcal{A}_{\tau_{0}}\left(\lambda_{1} i i^{\prime}\right)\left[\mathcal{L}_{J}\left(j \lambda i \mid j_{1} \lambda_{1} i_{1}\right) \mathcal{L}_{J}\left(j^{\prime} \lambda^{\prime} i^{\prime} ; j_{1} \lambda_{1} i_{2}\right)+\right. \\
\left.+\mathcal{L}_{J}\left(j^{\prime} \lambda^{\prime} i^{\prime} \mid j_{1} \lambda_{1} i_{1}\right) \mathcal{L}_{J}\left(j \lambda i \mid j_{1} \lambda_{1} i_{2}\right)\right] .
\end{gathered}
$$

The quantities $\mathcal{L}_{J}\left(j \lambda i \mid j^{\prime} \lambda^{\prime} i^{\prime}\right), \mathcal{T}_{J}\left(j \lambda i \mid j^{\prime} \lambda^{\prime} i^{\prime}\right)$ and $\mathcal{R}_{J}\left(j \lambda i \mid j^{\prime} \lambda^{\prime} i^{\prime}\right)$ vanish if the Pauli principle is not respected.

\section{Approximations}

\subsection{General}

As has been shown in [2] $\mathcal{L}_{J}$ are alternating quantities and their diagonal values are much greater than the nondiagonal ones. This is natural from the physical point of view as the Pauli principle is violated most probably in the configurations formed by identical quasiparticles. The same applies for the new quantities $\mathcal{T}_{J}$.

$$
\begin{aligned}
& \mathcal{L}_{J}\left(j \lambda i \mid j^{\prime} \lambda^{\prime} i^{\prime}\right)=\mathcal{L}(J j \lambda i) \delta_{j j^{\prime}} \delta_{\lambda \lambda^{\prime}} \delta_{i i^{\prime}}, \\
& \mathcal{T}_{J}\left(j \lambda i \mid j^{\prime} \lambda^{\prime} i^{\prime}\right)=\mathcal{T}(J j \lambda i) \delta_{j j^{\prime}} \delta_{\lambda \lambda^{\prime}} \delta_{i i^{\prime}},
\end{aligned}
$$

where

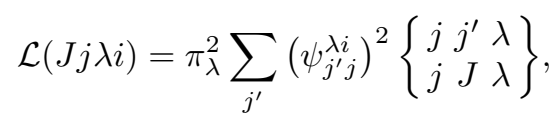




$$
\mathcal{T}(J j \lambda i)=\pi_{\lambda}^{2} \sum_{j^{\prime}} \psi_{j^{\prime} j}^{\lambda i} \varphi_{j^{\prime} j}^{\lambda i}\left\{\begin{array}{lll}
j & j^{\prime} & \lambda \\
j & J & \lambda
\end{array}\right\} .
$$

In this approximation

$$
V(J j \lambda i)=-\frac{1}{\sqrt{2}}(1+\mathcal{L}(J j \lambda i)+\mathcal{T}(J j \lambda i)) \Gamma(J j \lambda i) .
$$

The vertice $V$ is renormalized by the factor $(1+\mathcal{L}(J j \lambda i)+\mathcal{T}(J j \lambda i))$. In configurations with strong Pauli principle violation the quantities $\mathcal{L}(J j \lambda i)$ go to -1 and the quantities $\mathcal{T}(J j \lambda i)$ go to 0 . The role of the term $\mathcal{T}(J j \lambda i)$ in the renormalization becomes more important as the phonon collectiveness increases.

$$
W(J j \lambda i)=-\frac{1}{4} \frac{\pi_{\lambda}}{\pi_{J}}(1+\mathcal{L}(J j \lambda i)-\mathcal{L}(j J \lambda i)) \sum_{i^{\prime} \tau_{0}} \mathcal{A}_{\tau_{0}}\left(\lambda i i^{\prime}\right) \varphi_{J j}^{\lambda i^{\prime}} .
$$

As with the vertices $V$, the vertices $W$ are renormalized now by the factor $(1+\mathcal{L}(J j \lambda i)-\mathcal{L}(j J \lambda i))$ and again in configurations with strong Pauli principle violation the quantities $\mathcal{L}(J j \lambda i)$ go to -1 and the quantities $\mathcal{L}(j J \lambda i)$ go to 0 . The results for $V$ and $W$ show that configurations with $\mathcal{L}(J j \lambda i)$ close to -1 must be excluded from the configuration space.

$$
\begin{gathered}
K_{J}\left(j \lambda i j^{\prime} \lambda^{\prime} i^{\prime}\right)=\delta_{j j^{\prime}} \delta_{\lambda \lambda^{\prime}} \delta_{i i^{\prime}}(1+\mathcal{L}(J j \lambda i))\left(\omega_{\lambda i}+\varepsilon_{j}-\mathcal{R}(J j \lambda i)\right), \\
\mathcal{R}(J j \lambda i)=\frac{\mathcal{R}_{J}(j \lambda i \mid j \lambda i)}{1+\mathcal{L}(J j \lambda i)} .
\end{gathered}
$$

The quantities $\mathcal{R}(J j \lambda i)$ play a very important role as they shift the values of the poles and this shift depends on the extent of the Pauli principle violation [2].

Neglecting $G_{J}$ and $S_{J}\left(j \lambda i \mid j^{\prime} \lambda^{\prime} i^{\prime}\right)$, we arrive at the system of equations [6]

$$
\left[\left(\begin{array}{cc}
\varepsilon_{J} & 0 \\
0 & -\varepsilon_{J}
\end{array}\right)+\left(\begin{array}{cc}
M_{11} & M_{12} \\
M_{21} & M_{22}
\end{array}\right)\right]\left(\begin{array}{c}
C_{J \nu} \\
-E_{J \nu}
\end{array}\right)=\eta_{J \nu}\left(\begin{array}{c}
C_{J \nu} \\
-E_{J \nu}
\end{array}\right),
$$

where

$$
\begin{gathered}
M_{11}=\sum_{j \lambda i} \frac{1}{(1+\mathcal{L}(J j \lambda i))}\left(\frac{V^{2}(J j \lambda i)}{\eta_{J \nu}-\left(\omega_{\lambda i}+\varepsilon_{j}-\mathcal{R}(J j \lambda i)\right)}+\right. \\
\left.+\frac{W^{2}(J j \lambda i)}{\eta_{J \nu}+\omega_{\lambda i}+\varepsilon_{j}-\mathcal{R}(J j \lambda i)}\right), \\
M_{22}=\sum_{j \lambda i} \frac{1}{(1+\mathcal{L}(J j \lambda i))}\left(\frac{W^{2}(J j \lambda i)}{\eta_{J \nu}-\left(\omega_{\lambda i}+\varepsilon_{j}-\mathcal{R}(J j \lambda i)\right)}+\right. \\
\left.+\frac{V^{2}(J j \lambda i)}{\eta_{J \nu}+\omega_{\lambda i}+\varepsilon_{j}-\mathcal{R}(J j \lambda i)}\right),
\end{gathered}
$$




$$
\begin{aligned}
M_{12}=M_{21}=\sum_{j \lambda i} \frac{V(J j \lambda i) W(J j \lambda i)}{(1+\mathcal{L}(J j \lambda i))}( & \frac{1}{\eta_{J \nu}+\omega_{\lambda i}+\varepsilon_{j}-\mathcal{R}(J j \lambda i)}- \\
& \left.-\frac{1}{\eta_{J \nu}-\left(\omega_{\lambda i}+\varepsilon_{j}-\mathcal{R}(J j \lambda i)\right)}\right),
\end{aligned}
$$

leading to the equation

$$
M_{12} M_{21}=\left(\varepsilon_{J}+M_{11}-\eta_{J \nu}\right)\left(M_{22}-\varepsilon_{J}-\eta_{J \nu}\right) .
$$

\subsection{Limit Cases and Analisys}

The equation (6) can be approximated by the following one

$$
\varepsilon_{J}-\eta_{J \nu} \approx-M_{11}-\frac{M_{12}^{2}}{\left|2 \varepsilon_{J}-\left(M_{22}-M_{11}\right)\right|} .
$$

Therefore, neglecting the backward amplitudes, i.e. setting $W(J j \lambda i)=0$, (7) immediately reduces to the secular equation obtained earlier [2] :

$$
\varepsilon_{J}-\eta_{J \nu}=\sum_{j \lambda i} \frac{V^{2}(J j \lambda i)}{\left(\left(\omega_{\lambda i}+\varepsilon_{j}-\mathcal{R}(J j \lambda i)\right)-\eta_{J \nu}\right)(1+\mathcal{L}(J j \lambda i))} .
$$

The significant difference for the solution $\eta_{J}$ of the equation (7) as compared to the equation (8) comes from the second term in the r.h.s. of the expession (5) which contributes to a shift of the first solution of equation (7) to higher energies. The second term of the r.h.s. of equation (7) also contributes in the same direction but to a much smaller extent. The shift in energy becomes larger as the interaction between the quasiparticles and phonons increases. A critical value for the interaction exists as in (8) but now due to the second type of terms in $M_{11}$ an increase in the interaction leads to a shift of the first solution towards the pole as contrary to the case neglecting backward amplitudes where the solution moves in the opposite direction.

The inclusion of the matrix element $G_{J}$ in the system of equations can be accounted as a change of $M_{12}$ to $M_{12}+G_{J}$ but the resulting shift in the first solution turns out to be negligible.

\section{Numerical Results}

In order to give a qualitative picture of the effects on the structure of the low-lying states imposed by the backward-going amplitudes, numerical calculations for ${ }^{131} \mathrm{Ba}$ were performed. This isotope belongs to the transitional region where the anharmonic effects play a gradually increasing role at low and mainly at intermediate energies and therefore the results presented in this section may lack some accuracy 
because the wave function (2) does not contain configurations to account for these effects. The parameters of the Woods-Saxon potential are as follows:

Table 1. Parameters of the Woods-Saxon potential for ${ }^{131} \mathrm{Ba}$.

\begin{tabular}{|c|c|c|c|c|c|c|}
\hline$A$ & $N, Z$ & $r_{0}, \mathrm{fm}$ & $V_{0}, \mathrm{MeV}$ & $\kappa, \mathrm{fm}^{-2}$ & $\alpha, \mathrm{fm}^{-1}$ & $G_{N, Z}, \mathrm{MeV}$ \\
\hline 127 & $N=74$ & 1.280 & 43.40 & 0.413 & 1.613 & 0.124 \\
127 & $Z=53$ & 1.240 & 59.72 & 0.350 & 1.587 & 0.130 \\
\hline
\end{tabular}

Our study shows that in realistic calculations one must include phonons with $\lambda=2,3,4,5$. The strength parameters $\kappa^{(\lambda)}$ are adjusted so that the odd energy spectrum of the low-lying states is reasonably close to the experimental values. As a result, the energy of the first quadrupole state of ${ }^{130} \mathrm{Ba}$ has a value that is much higher than the experimental one.

Solving the systems of equations (4), one can find the structure of the wave functions (2) and the energies of the excited states. Working in a diagonal approximation for $\mathcal{L}_{J}$ and $\mathcal{T}_{J}$ this system reduces to a generalized eigenvalue problem. In Figure $1 \mathrm{a}$ comparison between the experimental values of the energies and the

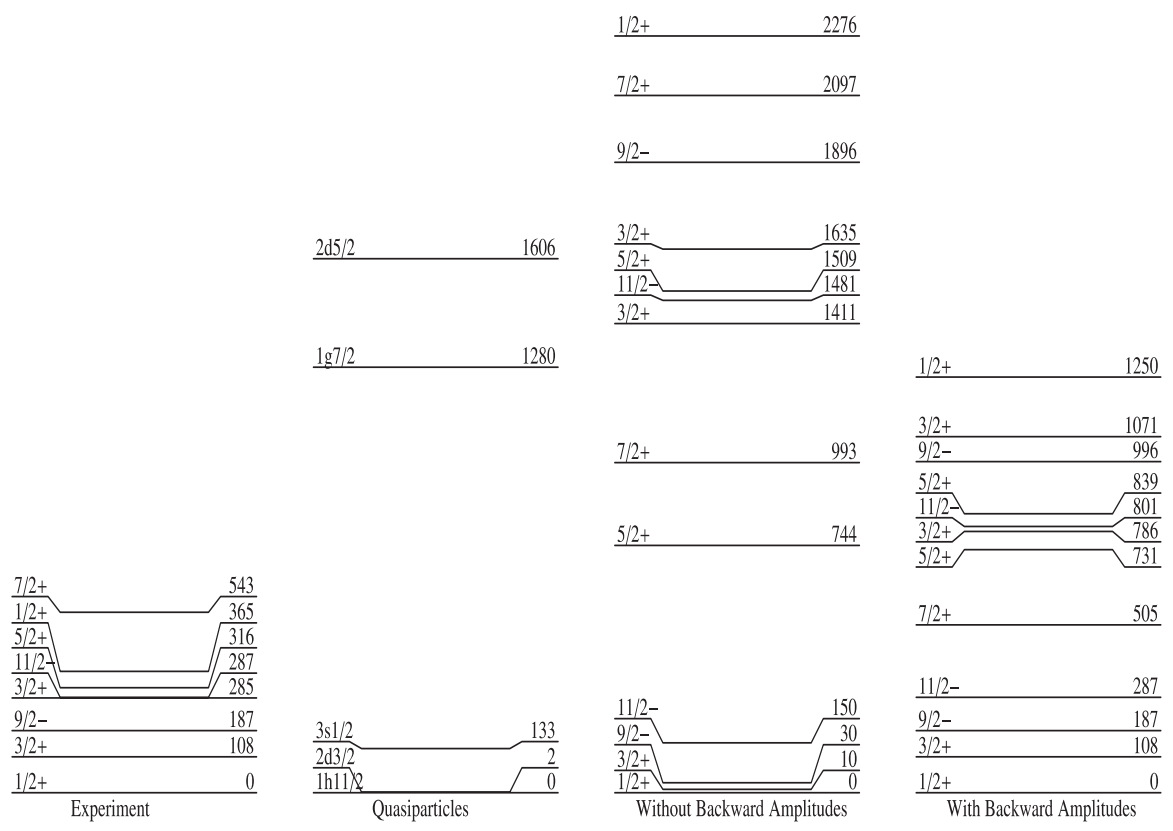

Figure 1. Low-lying energy spectrum of ${ }^{131} \mathrm{Ba}$ (in $\mathrm{KeV}$ ). The first column gives the experimental values [9], the second is the unperturbed 1qp spectrum, the third gives the levels resulting from the solution of (8), the fourth is the full calculation with backward amplitudes and Pauli principle corrections. 
theoretical calculations is presented. We restrict the calculation to the six neutron states $1 / 2^{+}, 3 / 2^{+}, 9 / 2^{-}, 11 / 2^{-}, 5 / 2^{+}, 7 / 2^{+}$. The level ordering presented in the third column on this figure generally agrees with the one obtained in [5]. The results clearly support the conclusion following from (7) as the first solutions obtained after the inclusion of the backward-going terms become closer to the first poles and consequently closer to the second solution thus significantly reducing the gap between the first $1 / 2^{+}$and the second $1 / 2^{+}$states as well as between the first $3 / 2^{+}$ and the second $3 / 2^{+}$states. The intruder state $9 / 2^{-}$deserves a special attention. The wave function of this state is practically a pure quasiparticle $\bigotimes$ phonon state with a structure $\left[1 h_{11 / 2} \otimes 2_{1}^{+}\right]_{9 / 2^{-}}$. The significant reduction of the energy of this state is due to the Pauli principle correction, the inclusion of which is essential for the correct ordering of the first several levels. Along with the experimental energies our calculations provide a reasonable description of the spectroscopic factors for the $(d, p)$-reactions: for the states $1 / 2+$ and $3 / 2+$ having experimentally measured values of the spectroscopic factors of 0.53 and 1.03 respectively, our calculations give 0.46 and 1.36 .

\section{Conclusion}

The comparison between theoretical calculations and experimental data for ${ }^{131} \mathrm{Ba}$ has shown that in order to describe the structure of the low-lying states in odd-mass nuclei far from the magic numbers one needs to take into account the Pauli principle and the ground state correlations effects simultaneously. Calculations for other Ba isotopes are in progress now. To improve this approach a self-consistent description of the mean field with more realistic effective nucleon-nucleon forces is desirable.

\section{References}

1. V. G. Soloviev, Theory of Atomic Nuclei: Quasiparticles and Phonons (Institute of Physics, Bristol and Philadelphia, 1992).

2. Chan Zuy Khuong, V. G. Soloviev, and V. V. Voronov, J. Phys. G: Nucl. Phys. 7, 151 (1981).

3. A. I. Vdovin, V. V. Voronov, V. G. Soloviev, and Ch. Stoyanov, Particles and Nuclei 16, 245 (1985).

4. S. Gales, Ch. Stoyanov, and A. I. Vdovin, Phys. Rep. 166, 125 (1988).

5. B. A. Alikov, K. M. Muminov, R. G. Nazmitdinov, and Chan Zuy Khuong, Izv. Akad. Nauk SSSR (ser. fiz.) 45, 2112 (1981).

6. V. Van der Sluys, D. Van Neck, M. Waroquier, and J. Ryckebusch, Nucl. Phys. A 551, 210 (1993).

7. D. Rowe, Nuclear collective motion (Menthuen, London, 1970).

8. A. Bohr and B. Mottelson, Nuclear Structure, vol. 2 (Benjamin, New York, 1975).

9. Nuclear Data Sheets 72, 487 (1994). 